\section{Effects of rotating backgrounds upon the perception of verticality*}

\author{
PHILIP C. HUGHES**, GERHARD A. BRECHER† \\ and \\ STEVEN M. FISHKIN†† \\ University of Oklahoma Medical Center, Oklahoma City, Oklahoma 73104
}

The effect of various types (sandpaper, spoke, spiral, and white) and speeds $(0,1 / 2,3,6,12$, and $30 \mathrm{rpm})$ of rotating disk backgrounds upon $276 \mathrm{Ss}$ ' estimates of the apparent verticality of a line moved toward physical verticality in either a clockwise or counterclockwise direction was investigated. The general finding was that the estimate of verticality was displaced away from pretest judgments in the direction of the disk rotation. The speed of rotation was significant with maximal vertical displacement at $6 \mathrm{rpm}$. The direction of line movement was significant with the result that maximal vertical displacement occurred when the line was moved in opposition to the direction of disk rotation. The type of disk employed made a significant difference in the estimates only when the line movement was opposite from the direction of disk rotation. Disks with inadequate stimulus contours (white) produced no significant effect. Results are discussed in terms of stimulus contour and ocular torsion.

Numerous studies have demonstrated that one's perception of verticality is affected by $S$ variables such as body position and individual differences (Witkin \& Asch, 1948).

Several investigators have also found that the perception of verticality is influenced by background stimulus conditions. Most of this work has focused on the visual frame surrounding an adjustable rod (Witkin \& Asch, 1948). One E (Brecher, 1934) has found that rotational movement of the visible background affects phenomenal verticality. The aim of the present study is to extend Brecher's work by examining the effect of various types and speeds of rotating backgrounds upon the perception of verticality.

\section{Subjects}

The Ss were 276 students from introductory psychology classes, with 216 serving in the basic factorial study (Experiment 1) and the remaining 60 Ss serving in two additional experiments (Experiments 2 and 3 ).

* This research was supported in part by USPHS Grant MH 10322 from the National Institute of Mental Health.

* Address: Department of Psychiatry and Behavioral Sciences, University of Oklahoma Medical Center, 800 Northeast Thirteenth Street, Oklahoma City, Oklahomn 73104.

TAddress: Department of Physiology and Biophysics, University of Oklahoma Medical Center, 800 Northeast Thirteenth Street, Oklahoma City, Oklahoma 73104.

+tAddress: Department of Psychiatry, University of Oklahoma Medical Center, 800 Northeast Thirteenth Street, Oklahoma City, Oklahoma 73104: Behavioral Science Laboratories, V.A. Hospital, 921 Northeast Thirteenth street, Oklahoma City, Thirteenth 73104 .

\section{Apparatus}

The apparatus consisted of a biting board, headrest, and aperture $(2.5 \mathrm{~cm}$ diam) which, in conjunction with a reduction screen, allowed the $S$ to view the stimulus display with his right eye only. The stimulus display was approximately $76 \mathrm{~cm}$ from the eye and consisted of a circular 24.8-cm-diam disk which was white, sandpaper, a disk of 16 black and white spokes, or a black and white spiral (five turns arithmetic, thick; Holland, 1965). The disks were rotated by synchronous motors at specified speeds. A 2-mm-thick white line was placed just in front of the disk. The line could be manually rotated with the center of rotation being coincident with the center point of the disk. The angle of the line with respect to physical verticality was determined by a scale placed on the apparatus. The scale was calibrated in quarter degrees and could not be seen by the $S$.

\section{Design and Procedure}

The main experiment was a 3 by 6 by 2 factorial. The first factor was the three stimulus disks: sandpaper, spokes, and spiral. The six levels of the second factor were the speeds of disk rotation: $0,1 / 2,3,6,12$, and $30 \mathrm{rpm}$. Factor 3 was the direction in which the line was moved during the verticality judgments. This was a repeated measures variable and represented a clockwise (CW) movement toward verticality on half the trials and a counterclockwise (CCW) movement of the line on other trials. Twelve Ss served in each of the 18 cells created by the combination of Factors 1 and 2.

The procedure involved instructing
Ss that they were to judge when a line appeared to them to be vertical as it was gradually moved toward physical verticality by $E$. Rate of line rotation was approximately $15 \mathrm{deg}$ of arc per 12 sec. The Ss were instructed and reminded during each trial to keep their right eyes fixated upon the center point of the disk. The biting board served to keep the head stationary. The Ss' first estimates were used, and they were not permitted to change their estimates during each of the separate trials.

Each $S$ went through three phases, a pre, a test, and a post phase, with two trials per phase. The prephase involved having the $S_{s}$ judge when the line appeared to be vertical when placed before a stationary white disk. Ss made one judgment with the line being moved toward the vertical in a $\mathrm{CW}$ direction and one in a $\mathrm{CCW}$ direction. The presentation of the starting directions was randomized. The starting positions of the line were between 12 and 24 deg from physical verticality, the actual values being determined on a random basis.

The second phase or test phase repeated the prephase procedure. However, in the test phase the stimulus disk was not white but was a sandpaper, spoke, or spiral disk which was either stationary or rotated $\mathbf{C W}$ at one of the following speeds: $1 / 2,3,6$, 12 , or $30 \mathrm{rpm}$.

The postphase, including the use of the stationary white disk, was identical with the prephase procedure. This phase served to check on any possible effects due to adaptation, order, and/or aftereffects. It was predicted that there would be little change in the judgments between the pre- and postphases, demonstrating the consistency of the measures employed.

Two additional experiments were run. In Experiment 2, $36 \mathrm{Ss}$ had their test disk rotate in a CCW direction rather than the $\mathrm{CW}$ direction used in the other experiments. This served as a further test on the significance of the direction of the line movement, answering the question of whether it is the absolute direction of line movement that is important (CW or CCW) or the line direction with reference to the direction of background movement, i.e., whether it is opposed to or in the same direction as the background movement. In Experiment 3, a group of $24 \mathrm{Ss}$ viewed the white disk rather than one of the three test disks during their test phase. It either remained stationary $(0 \mathrm{rpm})$ or rotated at $6 \mathrm{rpm}$, the most effective rotation speed in producing vertical displacement. This experiment served to demonstrate the importance of stimulus disk contours in producing vertical displacement. 
Table 1

Summary of 3 by 6 by 2 Analysis of Variance of Difference Scores

\begin{tabular}{|c|c|c|c|c|}
\hline Source of Variation & SS & df & MS & $\mathbf{F}$ \\
\hline (Type of Disk) & 6.56 & 2 & 3.28 & \\
\hline (Speed of Rotation) & $\mathbf{2 2 9 . 8 5}$ & 5 & 45.97 & $13.88 t$ \\
\hline 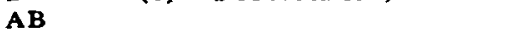 & 52.13 & 10 & 5.21 & 1.57 \\
\hline Ss Within Groups & 655.89 & 198 & 3.31 & \\
\hline C (Direction of Line Movement) & 157.39 & i & 157.39 & $46.30+$ \\
\hline ( & 31.61 & 2 & 15.39 & $4.65 *$ \\
\hline $\mathbf{B C}$ & 93.87 & $\overline{5}$ & 18.77 & $5.52 *$ \\
\hline $\mathbf{A B C}$ & 21.95 & 10 & 2.19 & \\
\hline C by Ss Within Groups & 672.94 & 198 & 3.40 & \\
\hline
\end{tabular}

$* p<.05 ;$ tp $<.001$
RESULTS AND DISCUSSION

The general direction of the effect observed under all conditions of Experiments 1 and 2, except when the background disk was stationary, was that the estimate of verticality made on the test judgments was displaced away from the pretest judgments in the direction of the disk rotation.

The data analyses for all experiments used difference scores in subtracted from test judgment scores of the same type. The pre and test judgments whose difference was calculated were ones in which the line was moved in the same direction, at approximately the same speed and from approximately the same starting position. Thus the only systematic difference between the two judgments was the presence during the test judgment of the (rotating) background disk. These difference scores were used to eliminate as potential sources of influence such factors as starting position effects and the stop-too-soon effect. The raw scores from which the difference scores were obtained represented the S's estimate of verticality. A minus raw score indicated the degrees to which the estimate was on the left or CCW side of the physical vertical, whereas a plus value represented an estimate to the right, or $\mathbf{C W}$, side. Physical verticality was assigned a zero value. Thus, a positive difference score would occur if the test judgment was $\mathrm{CW}$ in relation to the prejudgment. Table 1 shows the analysis of variance of the difference scores. The type of disk variable was not significant. Both speed of rotation and direction of line movement were gignificant at the .001 level.

The effect due to speed of rotation is interesting in that the speed producing maximum vertical shift is critical, with $6 \mathrm{rpm}$ being most effective and any increase or decrease from this speed being less effective. Figure 1 shows the effect of speed of background rotation separately for the two directions of line movement. Further research will be necessary to determine to what extent this speed of which prejudgment scores were considerably greater shift in the apparent vertical than did $\mathrm{CCW}$ line movement (mean difference score for $\mathrm{CW}=-3.39$ vs -1.08 for $\mathrm{CCW}$ ). The difference between means was significant at the .001 level $(t=4.98$, $\mathrm{df}=35$ ) and demonstrates that line movement in the direction opposite to maximal shift of the apparent vertical. It is clear, however, that although maximal shifts occur when line movement is opposite disk movement, disk movement also produces shifts in the apparent vertical. This is demonstrated by the fact that all four of the mean difference scores mentioned above $(\mathrm{CW}$ and $\mathrm{CCW}$ in Experiments 1 and 2), which includes those where line and disk move in the same direction, are significantly different from zero. (These effects were tested for significance in each case by testing the hypothesis that the number of positive difference scores $=$ number of negative difference scores = .5 . In all cases, the normal estimate of the binomial probability yielded $z$ scores $>2.66$, with $p<.01$ ).

The importance of the direction of line movement is also seen in the two significant interactions in Experiment 1. As indicated above, the direction of line movement was a significant source of variance. However, the effect of this variable is disk movement is associated with line movement in the same direction as line in the $\mathrm{CW}$ direction produced

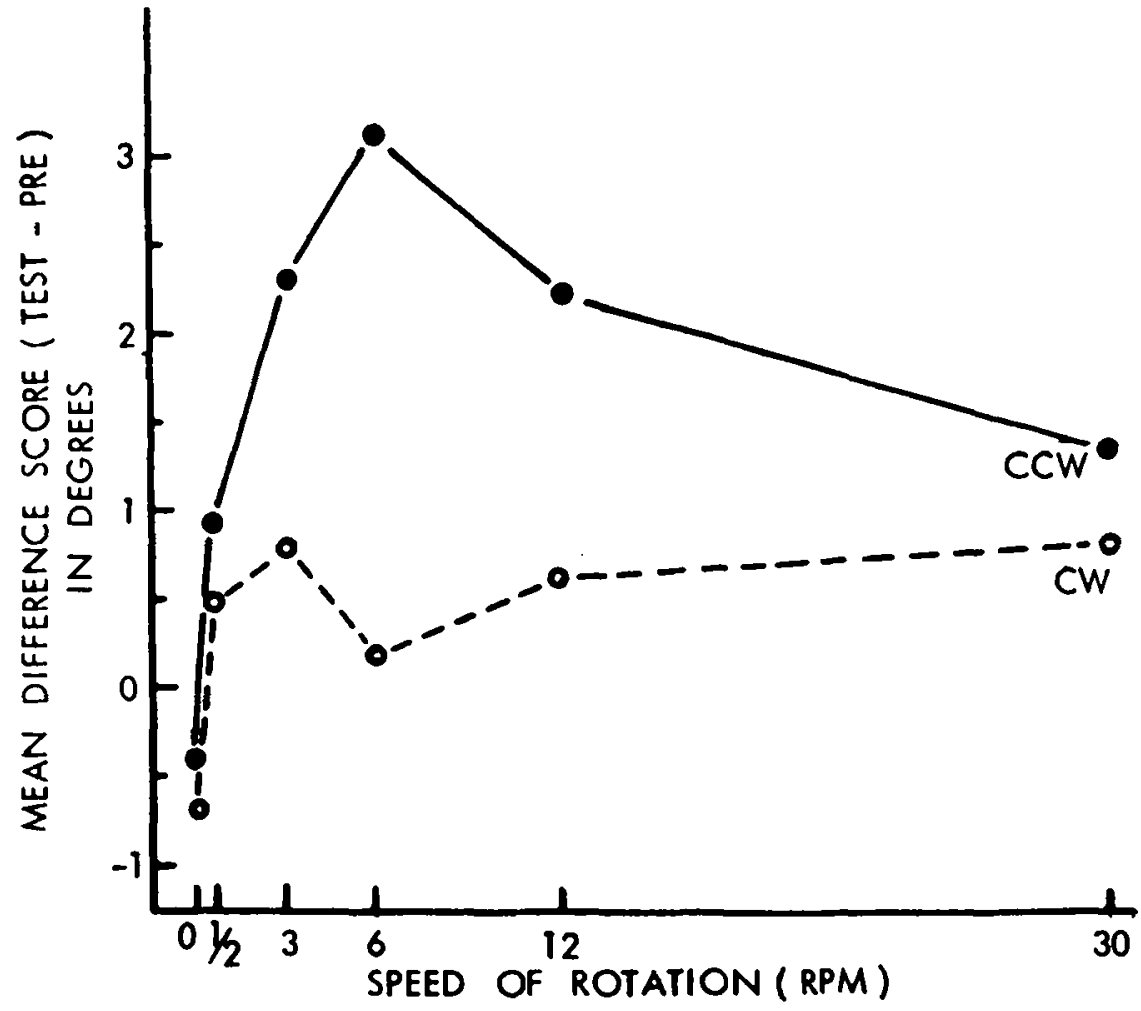

Fig. 1. Interaction of speed of rotation and direction of line movement. 


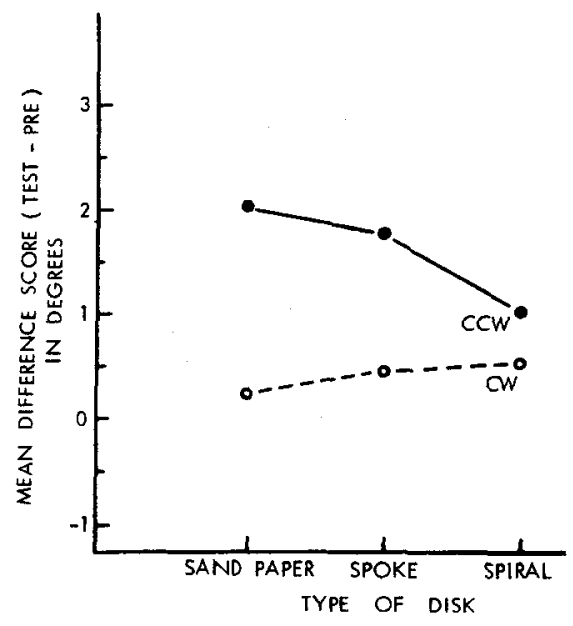

Fig. 2. Interaction of type of disk and direction of line movement.

complicated by its interaction with the two other variables in the experiment. The Type of Disk by Direction of Line Movement interaction (see Fig. 2) is in part due to a significant effect of type of disk when line movement is CCW but not when line movement is $\mathrm{CW}$. Simple main effect tests showed that with CCW line movement type of disk was significant, $\quad F(2,198)=4.9$, $\mathrm{p}<.01$, but with $\mathrm{CW}$ line movement the simple effect was not significant, $F(2,198)=0.4, p>.05$. Thus the type of disk makes a difference, but only when line movement is $\mathrm{CCW}$, i.e., in opposition to the direction of disk rotation.

The interaction between the direction of line movement and speed of rotation (see Fig. 1) shows that under the $\mathrm{CW}$ condition the various speeds of rotation apparently do not make a difference in verticality estimates, but with $\mathrm{CCW}$ line movement the speed of rotation does make a difference. Simple main effect tests showed that with CCW movement speed of rotation was significant, $\quad F(5,198)=15.89$, $\mathrm{p}<.001$, but with $\mathrm{CW}$ line movement it was not significant, $F(5,198)=2.22$, $p>.05$. It is interesting to note that in both interactions the $\mathrm{CW}$ condition, i.e., line movement in agreement with disk rotation, is relatively unaffected by the other two variables, whereas $\mathrm{CCW}$ line movement is affected by them.

The importance of stimulus contours is demonstrated in Experiment 3, in which Ss viewed either a white disk rotating at $6 \mathrm{rpm}$ during the test phase or a white disk which was stationary during the test phase. All Ss received both $\mathrm{CW}$ and CCW line movement. A 2 by 2 analysis of variance of difference scores showed that there was no significant difference between the stationary and 6-rpm conditions, $F(1,22)=4.02$, $p>.05$. In addition, the direction of line movement and the interaction of the two variables did not achieve significance. Thus, as predicted, rotation of background disks containing inadequate contours was not sufficient to produce displacement of apparent verticality. The results of Experiment 3 also minimize the possibility that suggested movement might be sufficient to produce the shift of apparent verticality. Such findings have been reported by Wapner and Werner (1957) in relation to other perceptual tasks. However, since the Ss in this condition were aware of the rotational movement of the background figure, it is unlikely that mere suggestion of movement in the absence of actual and adequate contour movement would be sufficient to produce the effect.

Correlated $t$ tests comparing the premeasures with the postmeasures for all Ss in Experiment 1 revealed no significant differences, the mean difference for the $\mathrm{CCW}$ measure being only $0.13 \mathrm{deg}$. Apparently temporal and order effects are not important in producing the results. Also, it would appear that the effects produced during the test phase are not strong enough or persistent enough or of the right type to produce "aftereffects" capable of influencing the posttest judgments. A Pearson $r$ on the pre- and postmeasures for $\mathrm{CW}$ and $\mathrm{CCW}$ scores resulted in highly significant correlations of .568 and .611 , respectively. These findings attest to the consistency of the measures employed and to the supposition that the significant differences are attributable only to the test phase conditions.

Additional work must be done before a clear understanding of the mechanisms involved in the obtained shifts in apparent verticality can be achieved. The work of Brecher (1934) suggests one mechanism which may be relevant. He found that, under conditions similar to those of the present experiment, the eye rotates in its socket in the same direction as the rotating background. To the extent that absolute orientation of the image on the retina is a factor in determining apparent verticality, ocular rotation implies that an altered physical orientation will appear vertical. In particular, if retinal points $A$ and $B$ are ordinarily stimulated when a physically vertical line is fixated, then those points would be stimulated by a line tilted in the direction of ocular rotation when the eye is rotated. This outcome corresponds to the direction of the results obtained in the present experiment. Further work in which ocular rotation is measured during judgments of verticality would obviously help to clarify the tenability of an ocular rotation explanation of the present results.

The results of Howard and Templeton (1964) appear to add an element of complexity to the eye-rotation explanation. They found that rotation of a line itself induces ocular rotation in the direction of line movement. On the assumption of additive effects, one would expect that when line and background rotate in the same direction maximal ocular rotation would occur, resulting in maximal displacement of the vertical. However, the present results show a stronger effect on apparent verticality when the test line was moved in the direction opposite to background rotation. An ocular rotation hypothesis might, however, still be tenable if the effect of opposite direction of rotation of background and line resulted in greater apparent rate of rotation of the dominant component of the visual field, i.e., the background disk. In the present experiment, however, no attempt was made to measure this type of contrast effect.

The importance of the type of stimulus background is indicated by the different results produced by the various disks when line rotation is in opposition to the disk rotation. No effect is apparent with a white contour-free disk, whereas the greatest effect is produced by a sandpaper disk. It is interesting to note that the magnitude of the shift of verticality increases with an aspect of the optical texture of the background figure. Thus, measurements of the background stimuli indicate that retinal elements were alternately stimulated by black and white sections of the background stimulus 2 times per revolution for the spiral figure and 16 times per revolution for the spoke figure. It is clear that the rate of alternate stimulation was considerably higher for the sandpaper disk. It does not seem unreasonable to suggest that ocular rotation might also be related to optical texture, such that finer textures (more rapid alternation during rotation) would be associated with greater ocular rotation.

The technique used in the present experiment has not permitted evaluation of the effects of background rotation which are independent of movement of the test line. In all cases, the line was moved either with or against the rotation of the background figure. Other psychophysical techniques could be used which involve the use of variously tilted stationary lines rather than a moving line. This approach would allow a more direct measure of the effects of various background factors upon the perception of verticality. 
REFERENCES

BRECHER, G. A. Die optokinetische Auslosung von Augenrollung und rotatorischem Nystagmus. I'flugers Archiv für die gesamte Physiologie des Menschen und der Tiere, 1934, 13-28.

HOLLAND, H. C. The spiral after-effect.
London: Pergamon Press, 1965.

HOWARD, I. P., \& TEMPLETON, W. B. Visually-induced eye torsion and tilt adaptation. Vision Research, 1964, 4, 433-437.

WAPNER, S., \& WERNER, H. Perceptual development: Worcester, Mass: Clark
University Press, 1957.

WITKIN, H A. \& ASCH, S. E. Studies in space orientation. Journal of Experimental Psychology, 1948, 38, 238.

(Accepted for publication May 17, 1971.) 